\title{
Neonatal and paediatric bloodstream infections: Pathogens, antimicrobial resistance patterns and prescribing practice at Khayelitsha District Hospital, Cape Town, South Africa
}

\author{
H Crichton, ${ }^{1} \mathrm{MB}$ ChB, DCH; N O'Connell, ${ }^{1} \mathrm{MB}$ ChB, FCPaed (SA), MMed (Paed); H Rabie, ${ }^{2} \mathrm{MB}$ ChB, FCPaed (SA), MMed, MSc (ID); \\ A C Whitelaw, ${ }^{3} \mathrm{MB}$ ChB, MSc, FCPath (SA) (Micro); A Dramowski, ${ }^{2}$ MB ChB, PhD, FCPaed (SA), MMed (Paed), Cert ID (SA) Paed, DCH \\ ${ }^{1}$ Department of Paediatrics, Khayelitsha District Hospital, Cape Town, South Africa \\ ${ }^{2}$ Department of Paediatrics and Child Health, Division of Paediatric Infectious Diseases, Faculty of Medicine and Health Sciences, Stellenbosch \\ University, Cape Town, South Africa \\ ${ }^{3}$ Division of Medical Microbiology, Department of Pathology, Faculty of Medicine and Health Sciences, Stellenbosch University; and National \\ Health Laboratory Service, Tygerberg Hospital, Cape Town, South Africa
}

Corresponding author: H Crichton (crchel002@gmail.com)

\begin{abstract}
Background. The epidemiology of neonatal and paediatric community-acquired and healthcare-associated bloodstream infections (BSI) at South African (SA) district hospitals is under-researched.

Objective. Retrospective review of neonatal and paediatric BSI (0 - 13 years) at Khayelitsha District Hospital, Cape Town, SA, over 3 years (1 March 2012 - 28 February 2015).

Methods. We used laboratory, hospital, patient and prescription data to determine BSI rates, blood culture yield and contamination rates, pathogen profile, antimicrobial resistance, patient demographics, BSI outcome and antibiotic prescribing practice.

Results. From 7427 blood cultures submitted, the pathogen yield was low $(2.1 \%, 156 / 7$ 427) while blood culture contamination rates were high (10.5\%, 782/7 427). Paediatric and neonatal BSI rates were 4.5 and 1.4/1 000 patient days, respectively. Gram-positive BSI predominated (59.3\%); Staphylococcus aureus (26.8\%) and Escherichia coli (21.6\%) were common pathogens. The median patient age was 3 months, with a predominance of males (57.7\%) and a $12.8 \%$ prevalence of HIV infection. Crude BSI-associated mortality was $7.1 \%(11 / 156)$, the death rate being higher in neonates than in infants and children $(6 / 40(15.0 \%)$ v. 5/116 (4.3\%), respectively; $p=0.03)$ and in patients with Gramnegative compared with Gram-positive bacteraemia (6/66 (9.1\%) v. 5/89 (5.6\%), respectively; $p=0.5)$. Most BSI episodes were communityacquired (138/156; 88.5\%), with high levels of extended-spectrum $\beta$-lactamase (ESBL) carriage among Klebsiella pneumoniae and E. coli isolates $(5 / 5(100 \%)$ and $8 / 33(24.2 \%)$, respectively). Antimicrobial management of BSI was inappropriate in $30.6 \%$ of cases (45/147), including incorrect empirical antibiotic (46.7\%), dual antibiotic cover (33.3\%) and inappropriately broad-spectrum antibiotic use (17.8\%). Conclusions. Antimicrobial-resistant pathogens (notably ESBL-producing Enterobacteriaceae) were common in community-acquired BSI. Paediatric clinicians at district hospitals require ongoing training in antibiotic stewardship and blood culture sampling.
\end{abstract}

S Afr Med J 2018;108(2):99-104. DOI:10.7196/SAMJ.2018.v108i2.12601

Bacterial bloodstream infections (BSI), both community acquired (CA) and healthcare associated (HA), are an important cause of neonatal and paediatric morbidity and mortality worldwide. ${ }^{[1]} \mathrm{A}$ meta-analysis of CA-BSI data from Africa in paediatric and adult patients established that non-typhoidal Salmonella, Escherichia coli, Staphylococcus aureus and Streptococcus pneumoniae are major pathogens, with CA-BSI associated mortality of $18 \%$ and a blood culture pathogen yield of $8.2 \%{ }^{[2]}$ Data on HA neonatal and paediatric BSI (HA-BSI) in Africa are scant, but confirm high case fatality, a predominance of Klebsiella pneumoniae, E. coli and S. aureus, and high levels of antimicrobial resistance (AMR) among nosocomial pathogens. ${ }^{[3,4]}$

The increasing frequency of AMR among BSI pathogens is of great concern for African countries, where access to care and broadspectrum antimicrobials is often limited. ${ }^{[5-8]}$ Important resistance phenotypes in BSI isolates include extended-spectrum $\beta$-lactamases (ESBL) and carbapenemase-producing Enterobacteriaceae. Data from a cross-sectional study at a South African (SA) children's hospital showed the presence of ESBL in $83 \%$ of K. pneumoniae isolates, ${ }^{[9]}$ along with the more recent emergence of carbapenem resistance due to carbapenemases. ${ }^{[10]}$ Common carbapenemases include New Delhi metallo- $\beta$-lactamase-1 (NDM-1) and OXA-48-like enzymes. ${ }^{[1]}$ The widespread and uncontrolled use of cephalosporins has contributed to the increased prevalence of ESBL-producing organisms, which may lead to increased BSI mortality, longer hospital stays and increased healthcare costs. ${ }^{[12-14]}$

The changing spectrum of pathogens, AMR patterns and clinical impact necessitates ongoing auditing, monitoring and publication of data on local pathogen profiles and antibiotic sensitivity patterns to inform clinical management and infection prevention efforts. ${ }^{[6,13,14]}$ For neonates and children admitted to SA district hospitals, research on the epidemiology and AMR patterns of CA- and HA-BSI will inform development of appropriate empirical antibiotic recommendations. Furthermore, there are very limited data on paediatric clinicians' prescribing practices to inform antimicrobial stewardship programmes at district hospitals.

\section{Objective}

To investigate the epidemiology of neonatal and paediatric BSI at Khayelitsha District Hospital (KDH) in Cape Town, SA, reporting 
BSI rates, pathogen profile, AMR patterns, risk factors for BSIassociated mortality and antibiotic prescribing practice.

\section{Methods \\ Setting}

KDH serves an urban community of $\sim 500000$ people. The population is young (one-third $<15$ years of age) and mainly black African (99\%), and most live in poor socioeconomic circumstances. ${ }^{[15]}$ The hospital's paediatric service comprises a 32-bed paediatric ward, a 6-bed paediatric short-stay ward in the emergency centre, a 12-bed neonatal unit and a 10-bed kangaroo mother care (KMC) unit. Patients are admitted from the community via the emergency centre or by referral through a network of primary care providers known as community health clinics. Moderately and critically ill children are referred to tertiary-level hospitals in the Cape metropolitan region.

\section{Investigation and management of BSI}

Blood cultures are obtained from neonates and children presenting with clinical signs and/or risk factors for severe bacterial infection (including fever, lethargy, shock, meningism, pneumonia, HIV infection and severe acute malnutrition) or following clinical deterioration during hospitalisation with suspected healthcare-associated infection (HAI). Blood cultures performed at $\mathrm{KDH}$ are processed at the National Health Laboratory Service (NHLS) microbiology laboratory at Tygerberg Hospital, using the automated BacT/Alert system (BioMerieux, France). Positive cultures were identified using standard microbiological methods, and susceptibility testing was performed using either the standard Kirby-Bauer disc diffusion method or the automated Vitek 2 AES (BioMerieux, France). Susceptibility results were interpreted using Clinical and Laboratory Standards Institute criteria. Empirical antibiotic recommendations for CA severe bacterial infections include a third-generation cephalosporin (cefotaxime or ceftriaxone) for suspected meningitis and ampicillin for lower respiratory tract infections; gentamicin is added to ampicillin for infants and immunocompromised patients. Neonates receive penicillin $G$ and gentamicin as first-line therapy or cefotaxime and ampicillin if meningitis is suspected. Empirical treatment for HA-BSI is piperacillin-tazobactam and amikacin, with escalation to meropenem in cases of suspected meningitis and in neonates and paediatric patients requiring transfer to tertiary care/an intensive care unit (ICU).

\section{Data extraction and analysis}

We retrospectively reviewed paediatric and neonatal BSI episodes at KDH between 1 March 2012 (when the hospital first opened) and 28 February 2015 using laboratory records, hospital data, patient folders and antimicrobial prescription charts to determine BSI rates, blood culture yield and contamination rates, pathogen profile, AMR rates, patient demographics, BSI outcome and antibiotic prescribing practice. The total number of blood cultures submitted during the study period was determined, with extraction of all positive blood cultures from children $(0-13$ years) from the NHLS electronic database. Inpatient days for the paediatric, neonatal, KMC and shortstay wards were obtained from the hospital data manager.

All positive blood culture isolates were classified as either pathogens or contaminants using the Centers for Disease Control and Prevention criteria. ${ }^{[16]}$ Positive blood cultures isolating the same pathogen within 14 days were considered a single episode of BSI. Similarly, positive blood cultures with a pathogen and a contaminant were considered a single episode of BSI. In vitro susceptibility of pathogens was determined from the NHLS records, and AMR phenotypes were described using proposed criteria. ${ }^{[17]}$
Isolates of E. coli and K. pneumoniae with reduced susceptibility to cefotaxime and/or ceftazidime were regarded as ESBL-producing organisms. No molecular tests were performed to confirm this, since these were retrospective data; however, plasmid-mediated ampC-like $\beta$-lactamases are very unusual in these organisms.

Positive blood cultures sampled $<48$ hours after hospital admission were considered to represent CA-BSI, whereas those obtained $>48$ hours after admission were classified as HA-BSI. Patients referred from a long-term healthcare institution or readmissions within 30 days of discharge were classified as HA-BSI. BSI and blood culture contamination rates were determined, with $95 \%$ confidence intervals (CIs). Demographic data were obtained from the laboratory records and hospital folder review, including age, weight, HIV status, antibiotic prescription record and outcome of hospitalisation. We calculated weight-for-age $z$-scores (WAZ) using World Health Organization anthropometric data and defined normal weight for age as $W A Z \geq 0$, underweight for age as $-2-0$, moderately underweight as $-2--3$ and severely underweight as $<-3$.

Where patient folders could be located, antibiotic prescriptions for the BSI episode were systematically reviewed by the authors (who include paediatricians, paediatric infectious diseases specialists and a microbiologist). Appropriateness of the empirical and targeted antibiotic prescriptions and compliance with local antibiotic recommendations were evaluated by the authors to achieve consensus regarding the appropriateness of each patient's empirical and targeted antibiotic prescription/s. Prescriptions were deemed appropriate if they were adequate to treat the patient's clinical diagnosis and in compliance with local treatment guidelines. Prescriptions were considered inappropriate if they were unsuitable to treat the patient's clinical diagnosis or in contravention of local treatment guidelines. Inappropriate prescriptions were subcategorised as follows: incorrect empirical antibiotic choice, dual antibiotic cover, and failure to use the most narrow-spectrum antibiotic available.

\section{Statistical analysis}

The BSI rate was calculated by dividing the total number of BSI episodes by the total inpatient days over the 3 -year period. The pathogen rate and contamination rate were calculated by dividing the total number of pathogens and contaminants, respectively, by the total number of blood cultures submitted. Standard practice is for only a single blood culture bottle to be taken at each blood culture collection episode, and the total number of bottles therefore represented the total number of blood culture collection episodes. A $\chi^{2}$ test for linear trend was used to assess changes in neonatal and paediatric BSI rates over time. Continuous and categorical data were analysed using Student's $t$-tests and Fisher's exact tests $/ \chi^{2}$ tests, as appropriate. Stata statistical software version 13.1 (StataCorp, USA) was used. To determine factors associated with mortality from BSI, binary logistic regression analysis was performed. Factors with a univariate $p$-value of $<0.1$ were entered into the model. A $p$-value of $<0.05$ was considered statistically significant.

\section{Ethical approval}

Ethical approval and waiver of individual informed consent was obtained from the Human Health Research Ethics Committee of Stellenbosch University (ref. no. S13/09/171) and the KDH ethics committee (ref. no. WC_2015RP15_794).

\section{Results}

Over the 3-year study period, the culture positivity rate was $12.6 \%$ (938/7 427 blood cultures), including 156 BSI episodes (2.1\% pathogen yield) and 782 contaminated blood cultures (10.5\%); contaminants 
included coagulase-negative staphylococci $(n=561,71.7 \%)$, Micrococcus spp. $(n=70$, $9.0 \%)$, non-pathogenic streptococci $(n=38$, $4.9 \%)$, Corynebacterium spp. $(n=32,4.1 \%)$ and Bacillus spp. $(n=31,4.0 \%)$. Most contaminated blood cultures were submitted from the emergency centre (488/782, 62.4\%). The neonatal and paediatric BSI rates were $1.4 / 1000$ patient days $(95 \%$ CI $0.9-1.9)$ and 4.5/1 000 patient days (95\% CI 3.7 - 5.3), respectively, with no significant change over the study period (Fig. 1).
Most blood culture samples from the patients with the 156 BSI episodes were submitted from the emergency centre $(122 / 156,78.2 \%)$, followed by the neonatal nursery $(27 / 156,17.3 \%)$ and the paediatric ward $(7 / 156,4.5 \%)$. The majority of episodes were CA-BSI (138/156, 88.5\%), with 18 episodes (11.5\%) of HA-BSI. Most BSI episodes were monomicrobial (145/156, $92.9 \%)$, but 11 episodes $(7.1 \%)$ were polymicrobial, with two pathogens each (i.e. total pathogen yield of 167 BSI pathogens)

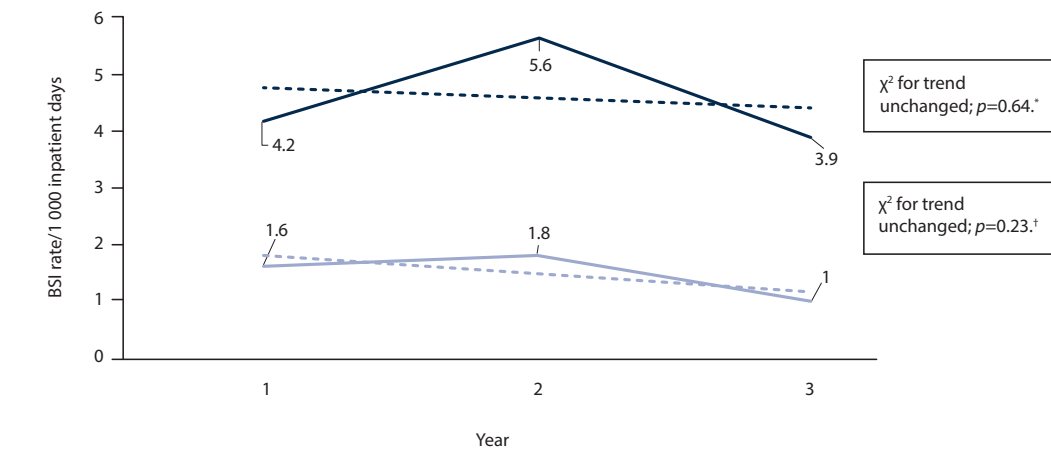

— BSI rate (paediatrics) — BSI rate (neonates) - - - - Linear BSI rate (paediatrics) - - - - Linear BSI rate (neonates)

Fig. 1. Trends in paediatric and neonatal BSI rates. $\left(B S I=\right.$ bloodstream infection; ${ }^{*}$ Paediatrics BSI rate year 1 (BSI/inpatient days $\times 1$ 000): 39/9 $234 \times 1$ 000; year 2: 50/3 $930 \times 1$ 000; year 340/10 $297 \times$ 1000 ; ${ }^{\dagger}$ Neonatal BSI rate year 1: 8/5 046 × 1000; year 2: $12 / 6705 \times 1$ 000; year 3: 7/7 504 × 1000.)
(Table 1). Of the 11 polymicrobial BSI episodes, two were in neonates and nine in children. Gram-positive bacteria were the predominant pathogen type isolated (99/167, 59.3\%), followed by Gramnegative pathogens $(67 / 167,40.1 \%)$, with only one fungal BSI pathogen. The most frequent pathogens in neonates $<28$ days of age $(n=40)$ were group B streptococci (20/40, 50.0\%), S. aureus (7/40,17.5\%) and E. coli $(6 / 40,15.0 \%)$. Among infants aged $>28$ days and children $(n=116)$, the most prevalent BSI pathogens were $S$. aureus (38/116, 32.8\%), E. coli $(30 / 116,25.9 \%)$ and S. pneumoniae (11/116, 9.5\%).

Patients with BSI had a median age of 3 months (interquartile range (IQR) 0.6 - 11 months) and a male predominance (57.7\%) (Table 2). HIV status was unknown in only 9.6\% (15/156); among the 141 patients with confirmed HIV status, the prevalence of HIV infection was $12.8 \%$ (18/141). Of the children $57.4 \%$ were HIV-exposed but uninfected (81/141). The most common BSI pathogens among HIV-infected children were $S$. pneumoniae $(n=5)$, followed by $E$. coli and S. aureus. The most prevalent comorbid conditions included prematurity (19.2\%), malnutrition (severely underweight) (10.3\%), tuberculosis (5.6\%) and cerebral palsy (3.0\%).

\begin{tabular}{|c|c|c|c|c|c|c|}
\hline \multirow[b]{2}{*}{ BSI episodes } & \multicolumn{6}{|c|}{ Pathogens isolated from 156 BSI episodes } \\
\hline & $\begin{array}{l}\text { Isolates, } n \\
(N=167)\end{array}$ & $\begin{array}{l}\begin{array}{l}\% \text { of total BSI } \\
\text { organisms* } \\
(N=167)\end{array} \\
\end{array}$ & $\%$ of group ${ }^{\dagger}$ & $\begin{array}{l}\% \text { of } \\
\text { neonates } \\
(N=40)\end{array}$ & $\begin{array}{l}\%>28 \\
\text { days of age } \\
(N=116)\end{array}$ & $\begin{array}{l}\text { \% of deaths } \\
(N=11)\end{array}$ \\
\hline Gram-negative organisms & 67 & & & & & \\
\hline \multicolumn{7}{|l|}{ Enterobacteriaceae } \\
\hline Escherichia coli & 36 & 21.6 & 53.6 & 15.0 & 25.9 & 27.3 \\
\hline Klebsiella pneumoniae & 9 & 5.4 & 13.4 & 7.5 & 5.2 & 18.1 \\
\hline Salmonella non-typhi & 4 & 2.4 & 6.0 & 0 & 3.4 & 0 \\
\hline Other & 5 & 3.0 & 7.5 & 5.0 & 2.6 & 0 \\
\hline Non-fermenting Gram-negative bacilli ${ }^{\varsigma}$ & 5 & 3.0 & 7.5 & 0 & 4.3 & 0 \\
\hline \multicolumn{7}{|l|}{ Other } \\
\hline Neisseria meningitidis & 6 & 3.6 & 9.0 & 0 & 5.2 & 9.1 \\
\hline Haemophilus influenzae & 2 & 1.2 & 3.0 & 0 & 1.7 & 0 \\
\hline Gram-positive organisms & 99 & & & & & \\
\hline Staphylococcus aureus & 45 & 26.8 & 45.5 & 17.5 & 32.8 & 9.1 \\
\hline Streptococcus pneumoniae & 12 & 7.2 & 12.1 & 0 & 10.3 & 0 \\
\hline Group B streptococcus & 26 & 15.6 & 26.3 & 50.0 & 5.2 & 27.3 \\
\hline Enterococcus spp. & 10 & 6.0 & 10.1 & 5.0 & 6.9 & 9.1 \\
\hline Listeria monocytogenes & 2 & 1.2 & 2.0 & 5.0 & 0 & 0 \\
\hline Others & 4 & 2.4 & 4.0 & 0 & 3.4 & 0 \\
\hline Fungi & 1 & & & & & \\
\hline Candida parapsilosis & 1 & 0.6 & 100 & 0 & 0 & 0 \\
\hline \multicolumn{7}{|c|}{ 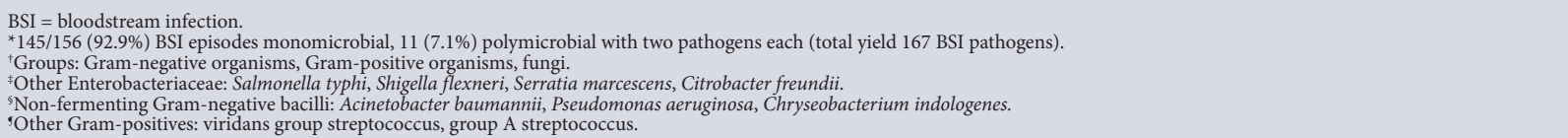 } \\
\hline
\end{tabular}




\begin{tabular}{|c|c|c|c|c|}
\hline Variable & LC-BSI $(N=156)$ & CA-BSI $(N=138)$ & HA-BSI $(N=18)$ & $p$-value \\
\hline Gender (male), $n(\%)$ & $90(57.7)$ & $78(56.5)$ & $12(66.7)$ & 0.41 \\
\hline Age (days), median (IQR) & $94(18-343)$ & $113(30-358)$ & $15(1-49)$ & 0.006 \\
\hline Age category, $n(\%)$ & & & & 0.05 \\
\hline$<28$ days & $40(25.6)$ & $31(22.5)$ & $9(50.0)$ & \\
\hline 28 - 364 days & $77(49.4)$ & $71(51.4)$ & $6(33.3)$ & \\
\hline $1-5$ years & $26(16.7)$ & $23(16.7)$ & $3(16.7)$ & \\
\hline$>5-13$ years & $13(8.3)$ & $13(9.4)$ & 0 & \\
\hline HIV status, $n(\%)$ & & & & 0.001 \\
\hline Infected & $18(11.5)$ & $16(11.6)$ & $2(11.1)$ & \\
\hline Negative, unexposed & $73(46.8)$ & $57(41.3)$ & $16(88.9)$ & \\
\hline Exposed, uninfected & $50(32.1)$ & $50(36.2)$ & 0 & \\
\hline Unknown & $15(9.6)$ & $15(10.9)$ & 0 & \\
\hline Ward at diagnosis, $n(\%)$ & & & & 0.001 \\
\hline Emergency unit & $122(78.2)$ & $117(84.8)$ & $5(27.8)$ & \\
\hline Paediatric ward & $7(4.5)$ & $6(4.3)$ & $1(5.6)$ & \\
\hline Neonatal nursery/KMC & $27(17.3)$ & $15(10.9)$ & $12(66.6)$ & \\
\hline Outcome, $n(\%)$ & & & & 0.47 \\
\hline Died & $11(7.0)$ & $9(6.5)$ & $2(11.1)$ & \\
\hline Survived & $145(93.0)$ & $129(93.5)$ & $16(88.9)$ & \\
\hline Impact, $n(\%)$ & & & & - \\
\hline District hospital & $70(44.9)$ & $63(45.7)$ & $7(38.9)$ & \\
\hline Tertiary ward & $59(37.8)$ & $51(37.0)$ & $8(44.4)$ & \\
\hline Transferred to ICU & $27(17.3)$ & $24(17.3)$ & $3(16.7)$ & \\
\hline Duration of hospitalisation (days), median (IQR) & $10(5-17)$ & $9(4-14$ & $21.5(17-26)$ & - \\
\hline Time to HA-BSI onset (days), median (IQR) & $\mathrm{n} / \mathrm{a}$ & $\mathrm{n} / \mathrm{a}$ & $5(2-9)$ & - \\
\hline
\end{tabular}

Eighteen episodes (11.5\%) of HA-BSI were documented, with a median hospital stay of 5 days (IQR 2 - 8) preceding HA-BSI onset. Ten HA-BSI episodes (55.6\%) occurred in the neonatal nursery among premature babies. HA-BSI pathogens were $K$. pneumoniae $(n=4)$, E. coli $(n=3)$, group B streptococci $(n=3)$, S. aureus $(n=2)$, Enterococcus spp. $(n=2)$, Pseudomonas aeruginosa $(n=1)$, S. pneumoniae $(n=1)$, Serratia marcescens $(n=1)$ and non-typhoidal Salmonella $(n=1)$. Two patients died following episodes of $E$. coli and E. faecalis HA-BSI, at a median of 34 days from BSI onset.

The clinical impact of the 156 BSI episodes was severe: 86 patients (55.1\%) required transfer to tertiary care, including 27 (17.3\%) who required ICU admission for ventilation and/or inotropic support. The overall crude in-hospital BSI-associated mortality rate was $7.1 \%(11 / 156)$, the case fatality rate being higher among neonates (0 - 28 days) compared with infants/older children (6/40 (15.0\%) v. $5 / 116$ (4.3\%), respectively; $p=0.03$ ). The median hospital stay from admission to death for patients with CA-BSI was 9 days (IQR 4 - 15). The BSI-associated crude mortality rate in HIV-infected children was higher than that in HIV-uninfected/HIV-unknown children (3/18 $(16.6 \%)$ v. $8 / 138(5.8 \%)$, respectively; $p=0.05)$. Mortality was higher among patients with Gram-negative BSI than among those with Gram-positive and fungal infections, but not significantly so (6/66 (9.1\%) v. $5 / 90(5.6 \%)$, respectively; $p=0.5)$. There was no difference in crude mortality rates between neonates/children with HA-BSI compared with CA-BSI $(2 / 18$ (11.1\%) v. 9/138 (6.5\%), respectively; $p=0.62$ ). On multivariate analysis, age $<28$ days and malnutrition (WAZ $<-3$ ) were significantly associated with mortality (odds ratio 8.6 (95\% CI 1.7 - 45.0) and 11.3 (95\% CI 1.7 - 74.0), respectively; $p=0.01$ ). Among the children who died, most deaths occurred after transfer to the ICU $(5 / 11,45.5 \%)$, followed by deaths in the emergency centre $(4 / 11,36.4 \%)$ and the neonatal nursery $(2 / 11$, $18.2 \%)$.

AMR rates were high for both CA-BSI and HA-BSI pathogens; among the CA-BSI pathogens, $8 / 33$ (24.2\%) E. coli and 5/5 (100\%) K. pneumoniae were ESBL producers. Folder review of the $13 \mathrm{CA}-$ ESBL BSI episodes found that only one of the 13 children had been admitted to hospital in the preceding 30 days, suggesting community acquisition of the ESBL-producing pathogens. Of the HA-BSI pathogens, all the K. pneumoniae $(n=4)$ but none of the E. coli were ESBL producers. BSI-associated crude mortality was significantly higher among children with CA-BSI pathogens that produced ESBLs v. all other CA-BSI pathogens (3/13 (23.1\%) v. 6/125 (4.8\%), respectively; $p=0.04)$. Of the 42 S. aureus isolates, $4(9.5 \%)$ were methicillin-resistant CA-BSI.

Meropenem provided greatest in vitro coverage for HA-BSI pathogens, with susceptibility in $16 / 18$ (88.9\%), followed by piperacillin-tazobactam $(14 / 18,77.8 \%)$ and amikacin $(7 / 18,38.9 \%)$. The institution's recommended regimen for HAI (piperacillintazobactam plus amikacin) provided good empirical coverage (16/18, 88.9\%). Of CA-BSI pathogens, $68.1 \%$ were susceptible (in vitro) to first-line antibiotics (ampicillin and/or gentamicin) and $69.6 \%$ were susceptible to ceftriaxone. No carbapenem-resistant Enterobacteriaceae, vancomycin-resistant enterococci or fluconazoleresistant Candida species were isolated.

For the 156 BSI episodes, 147 complete patient folders and prescription charts $(94.2 \%)$ were located for review. Nearly one-third of patients $(45 / 147,30.6 \%)$ were assessed through expert review as having received inappropriate antimicrobial therapy (empirical, targeted or both) (Table 3). The leading causes of inappropriate antibiotic use were incorrect empirical antibiotic choice $(21 / 45$, 
Table 3. Antimicrobial management of BSI $(N=147)^{*}$

\begin{tabular}{ll}
\hline & $n(\%)$ \\
\hline Appropriate & $102(69.4)$ \\
Inappropriate $^{\dagger}$ & $45(30.6)$ \\
Incorrect empirical antibiotic prescribed & $21 / 45(46.7)$ \\
Dual antibiotic cover & $15 / 45(33.3)$ \\
Narrowest-spectrum agent not used & $8 / 45(17.8)$ \\
Inadequate documentation & $7 / 45(15.6)$ \\
Incorrect antibiotic switch & $5 / 45(11.1)$ \\
Blood culture result not followed up & $4 / 45(8.9)$ \\
Second-line protocol not followed & $4 / 45(8.9)$ \\
Earlier oral switch indicated but not made & $2 / 45(4.4)$ \\
Stock shortages & $2 / 45(4.4)$ \\
Initial escalation of antibiotic therapy required & $1 / 45(2.2)$ \\
BSI = bloodstream infection. \\
*9 folders could not be retrieved or had incomplete antimicrobial prescription records. \\
'In some cass there was more than one reason for assessing antimicrobial management of \\
the BSI episode as inappropriate.
\end{tabular}

46.7\%), dual antibiotic cover $(15 / 45,33.3 \%)$ and failure to use the most narrow-spectrum antibiotic available $(8 / 45,17.8 \%)$.

\section{Discussion}

Our study documented pathogen yields markedly lower than reported from other African settings (8.2\%). ${ }^{[2]}$ Several factors, including district hospital level of care, antibiotics administered prior to admission and inadequate volumes of blood, may have contributed to the lower yield. According to the Integrated Management of Childhood Illness guidelines, ${ }^{[18]}$ any child who fulfils the criteria for serious illness should receive intramuscular ceftriaxone prior to transfer, which may contribute to the lower pathogen yield observed and may also contribute to the proportionately higher prevalence of ESBLs in CA Enterobacteriaceae.

Of great concern is the high rate of blood culture contamination identified, which in addition to creating clinical uncertainty has a financial impact on the hospital owing to the costs of further testing and management, as well as implications for the patient, who has to undergo further investigations and a prolonged hospital stay. The contamination rate was more than three times higher than the internationally accepted rate of $3 \%{ }^{[19]}$ This could be attributed to the difficulty in obtaining blood cultures from severely ill children, the busy emergency centre setting, staff and stock shortages, inadequate or irregular training of staff, high staff turnover, limited resources, and unfamiliarity with the blood culturing protocol. Factors known to contribute to increased blood culture contamination rates include young patient age ( $<36$ months) and inexperienced physicians (trainees v. specialists). ${ }^{[19,20]}$ Given the high contamination rates, urgent interventions such as regular training workshops on the correct sampling technique, feedback of monthly contamination rates to clinical staff, and intermittent checks of blood volume inoculum (by weighing culture bottles) should be implemented. In addition, all wards and the emergency centre should ensure that there is sufficient stock of sterile blood culture packs and consumables.

At our institution, Gram-positive BSI pathogens (59.3\%) predominated, particularly among CA-BSI, and in contrast to many African BSI studies reporting Gram-negative predominance. ${ }^{[2,21]}$ Gram-negative pathogens predominated in HA-BSI episodes, in keeping with findings of neonatal and paediatric BSI studies at two tertiary hospitals in Cape Town. ${ }^{[5,21,22]}$ A large proportion of BSI patients $(55.1 \%)$ required transfer to tertiary-level hospitals, which highlights the impact of BSI on children as well as the healthcare costs involved. Of note is the high number of CA ESBL organisms in our study, with a mortality rate three times higher than the overall crude mortality rate. Further scrutiny of CA ESBL BSI would have been beneficial in terms of determining the number of clinic visits with antibiotic administration prior to admission, and possible contributing factors leading to the emergence of resistance at a community level.

Knowledge of local organisms and their sensitivity profiles in a peripheral hospital is invaluable for development and revision of institutional antimicrobial guidelines. Regular review of AMR rates in neonatal and paediatric CA-BSI isolates will be required in view of the $68-70 \%$ current coverage of CA-BSI pathogens with empirical therapy (ampicillin/gentamicin or ceftriaxone). The institution's empirical recommendations for HA-BSI provided high in vitro coverage, although the number of BSI in this category was very low.

Inappropriate empirical antibiotic choice accounted for almost half $(46.7 \%)$ of the prescriptions that were evaluated as inappropriate. Incorrect initial antimicrobial choice has been associated with increased mortality in a study in SA ICUs (27\% v. $11 \%$ when antibiotic choice was apppropriate). ${ }^{[23]}$ In addition, 33.3\% of prescriptions had dual antibiotic cover, where antibiotics were added to treatment plans and prescription charts were not amended. Based on the many inappropriate prescribing practices identified in this study, we recommend ongoing antibiotic stewardship audits, pharmacy audits and regular antibiotic stewardship clinical ward rounds to improve prescriber awareness and patient outcomes at district hospitals. Assessment of clinicians' reasons for instituting, changing or deviating from recommended prescribing practice was problematic, owing to very poor documentation in the hospital records. Correct prescribing procedures need to be adhered to and documentation in clinical notes needs to be improved. At present, few structured antibiotic stewardship rounds are conducted in district-level hospitals owing to limited resources and expertise (i.e. lack of on-site microbiology, infectious diseases and/or clinical pharmacology personnel).

Similarly, improvements to documentation in clinical patient case notes should be highlighted to ensure continuity of care and assist in future audits. Healthcare professionals should take care to document the time of positive blood culture and the corresponding antibiotic choice. In the case of upscaling to second-line antibiotics, the clinical reason should be carefully documented. Prescription charts should be reviewed daily to ensure that all medications are correct and stopped timeously. The South African Antibiotic Stewardship programme prescription chart ${ }^{[24]}$ should be used at district-level hospitals to improve prescribing practices. This will aid judicious prescribing practices and improve antibiotic stewardship rounds and audits at a district level.

\section{Study limitations}

Our study has several limitations: a relatively small number of BSI episodes and short study duration, which limit the ability to draw conclusions regarding antimicrobial susceptibility and prescribing trends; inability to observe blood culture inoculum volume and sampling technique (which influence blood culture yield and contamination rates); and inability to document recent hospitalisation and previous antibiotic exposures as variables affecting classification of CA- v. HA-BSI and blood culture yield, respectively. We reported a crude BSI-associated in-hospital mortality rate, and owing to the retrospective nature of the study it was not possible to determine whether deaths were due to the BSI alone or attributable to another event. The median number of days from blood culture specimen collection to death in the CA-BSI (9 days) and HA-BSI cohorts (34 days) suggests that additional factors may have contributed to our patients' deaths. A further limitation was reliance 
on electronic records to evaluate antimicrobial prescription practices, with exclusion of nine records (5.8\%) that were incomplete. Frequent antibiotic stock shortages influenced prescribing practice and may have resulted in delayed institution of targeted antibiotic therapy or de-escalation to narrow-spectrum agents. Despite these limitations, we believe that this first description of the epidemiology, outcome and therapy of neonatal and paediatric BSI at a large urban SA district hospital has provided valuable data to inform quality improvement in blood culture sampling and antibiotic stewardship.

\section{Conclusions}

Neonatal and paediatric BSI patterns at this SA district hospital show a predominance of Gram-positive, community-acquired pathogens and concerning levels of ESBL carriage among CA Enterobacteriaceae. Ongoing training of paediatric clinicians is needed to address high rates of blood culture contamination and inappropriate antibiotic prescribing practices.

\section{Acknowledgements. None.}

Author contributions. All authors (HC, AD, ACW, NO'C, HR) contributed to the study design and critical review of the manuscript. All authors read and approved the final manuscript.

Funding. None.

Conflicts of interest. None.

1. Liu L, Johnson HL, Cousens S, et al. Global, regional and national causes of child mortality: An updated systemic analysis for 2010 with time trends since 2000. Lancet 2012;379(9832):2151-2161. https://doi.org/10.1016/S0140-6736(12)60560-1

2. Reddy EA, Shaw AV, Crump J. Community-acquired bloodstream infections in Africa: A systematic review and meta-analysis. Lancet 2010;10(6):417-432. https://doi.org/10.1016/S1473-3099(10)70072-4 3. Aiken AM, Mturi N, Niuguna P, et al.; Kilifi Bacteraemia Surveillance Group. Risk and causes of paediatric hospital-acquired bacteraemia in Kilifi District Hospital, Kenya: A prospective cohort study. Lancet 2011:378(9808):2021-2027. https://doi.org/10.1016/S0140-6736(11)61622-X

4. Dramowski A, Madide A, Bekker A. Neonatal nosocomial bloodstream infections at a referral hospital in a middle-income country: Burden, pathogens, antimicrobial resistance and mortality. Paediatr Int in a middle-income country: Burden, pathogens, antimicrobial resistance and mor

5. Morkel G, Bekker A, Marais BJ, Kirsten G, van Wyk J, Dramowski A. Bloodstream infections and Morkel G, Bekker A, Marais BJ, Kirsten G, van Wyk J, Dramowski A. Bloodstream infections and
antimicrobial resistance patterns in a South African neonatal intensive care unit. Paediatr Int Child antimicrobial resistance patterns in a South African neonatal intensive care
Health 2014;34(2):108-114. https://doi.org/10.1179/2046905513Y.0000000082

6. Kenyon CR, Fatti G, Schreuder N, Bonorchis K, Meintjies G. The value of blood culture audits at peripheral hospitals. S Afr Med J 2012;102(4):224-225
7. Mendelson M, Whitelaw A, Nicol M, Brink A. Wake up, South Africa! The antibiotic 'horse' has bolted. S Afr Med J 2012;102(7):607-608. https://doi.org/10.7196/SAMJ.5759

8. Hwang A, Gums I. The emergence and evolution of antimicrobial resistance: Impact on a global scale. 8. Hwang A, Gums J. The emergence and evolution of antimicrobial resistance: Impact on
Bioorg Med Chem 2016;24(24):6440-6445. https://doi.org/10.1016/j.bmc.2016.04.027

9. Buys H, Muloiwa R, Bamford C, Ely B. Klebsiella pneumoniae bloodstream infections at a South 9. Buys H, Muloiwa R, Bamford C, Ely B. Klebsiella pneumoniae bloodstream infections at a South
African children's hospital 2006 - 2011, a cross-sectional study. BMC Infect Dis 2016;16:570. https:// African children's hospital $2006-2011$
doi.org/10.1186/s12879-016-1919-y

10. Perovic O, Singh-Moodley A, Dusé A, et al. National sentinel site surveillance for antimicrobial resistance in Klebsiella pneumoniae isolates in South Africa, 2010 - 2012. S Afr Med J 2014;104(8):563568. https://doi.org/10.7196/SAMJ.7617

11. National Institute for Communicable Disease. Surveillance of antimicrobial resistance. Commun Dis Comm 2017;16(5a):7-10. http://www.nicd.ac.za/wp-content/uploads/2017/03/NICD-CommunicableDiseases-Communique_May2017_final2.pdf (accessed 4 December 2018).

12. Benner K, Prabhakaran P, Lowros AS. Epidemiology of infections due to extended-spectrum beta-lactamase-producing bacteria in a paediatric intensive care unit. J Pediatr Pharmacol Ther 2014;19(2):83-90. https://doi.org/10.5863/1551-6776-19.2.83

13. Ndir A, Diop A, Faye P, Cisse M, Ndoye B, Astagneau P. Epidemiology and burden of bloodstream infections caused by extended-spectrum beta-lactamase producing Enterobacteriaceae in a paediatric infections caused by extended-spectrum beta-lactamase producing Enterobacteriaceae in a paed
hospital in Senegal. PLoS One 2016;11(2):1-13. https://doi.org/10.1371/journal.pone.0.143729

14. Blomberg B, Manji KP, Urassa W, et al. Antimicrobial resistance predicts death in Tanzanian children with blood stream infection: A prospective cohort study. BMC Infect Dis 2007;43(7):1-14. https://doi. org/10.1186/1471-2334-7-43

15. City of Cape Town. 2011 Census Suburb Khayelitsha. 2013. http://resource.capetown.gov.za/ documentcentre/Documents/Mapsandstatistics/2011_Census_CT_Suburb_Khayelitsha_Profile.pdf (accessed 2 November 2016).

16. Centers for Disease Control and Prevention and National Healthcare Safety Network. Bloodstream infection event (central line-associated bloodstream infection and non-central line-associated bloodstream infection). www.cdc.gov/nhsn/pdfs/pscmanual/4psc_clabscurrent.pdf (accessed 2 November 2016)

17. Magiorakos AP, Srinivasan A, Carey RB, et al. Multidrug-resistant, extensively drug resistant and pandrug-resistant bacteria: An international expert proposal for interim standard definitions for acquired resistance. Clin Microbiol Infect 2012;18(3):268-281. https://doi.org/10.1111/j.14690691.2011.03570.x

18. National Department of Health, South Africa. Integrated Management of Childhood Illness Guidelines. 2014. paediatrics.ukzn.ac za/Files/2014\%20IMCI\%20CHART\%20BOOKLET\%20Final. pdf (accessed 26 April 2017)

19. Hall K, Lyman J. Updated review of blood culture contamination. Clin Microbiol Rev 2006;19(4):788802. https://doi.org/10.1128/CMR.00062-05

20. Pavlovsky M, Press J, Peled N, Yagupsky P. Blood culture contamination in paediatric patients: Young children and young doctors. Pediatr Infect Dis J 2006;25(7):611-614. https://doi.org/10.1097/01. inf.0000220228.01382.88

21. Lochan H, Bamford C, Eley B. Blood cultures in sick children. S Afr Med J 2013;103(12):918-920. https://doi.org/10.7196/SAMJ.6979

22. Dramowski A, Cotton M, Rabie H, Whitelaw A. Trends in paediatric bloodstream infections at a South African referral hospital. BMC Pediatr 2015;15(33):1-11. https://doi.org/10.1186/s12887-015-0354-3

23. Paruk F, Richards G, Scribante J, Bhagwanjee S, Mer M, Perrie H. Antibiotic prescription practices and their relationship to outcome in South African intensive care units: Findings of the prevalence of infection in South African Intensive Care Units (PISA) Study. S Afr Med J 2012;102(7):613-616. https://doi.org/10.7196/SAMJ.5833

24. Federation of Infectious Diseases Society of Southern Africa (FIDSSA), South African Antibiotic Stewardship Programme prescription chart. http://www.fidssa.co.za/SAASP/Prescription_Chart (accessed 2 November 2016).

Accepted 7 August 2017 